\title{
Le fondamental est-il l'essentiel ? (Aristote, Métaphysique Z 3 )
}

Rudolf Boehm

\section{Citer ce document / Cite this document :}

Boehm Rudolf. Le fondamental est-il l'essentiel ? (Aristote, Métaphysique Z 3). In: Revue Philosophique de Louvain. Troisième série, tome $64, n^{\circ} 83,1966$. pp. 373-389;

doi : https://doi.org/10.3406/phlou.1966.1335

https://www.persee.fr/doc/phlou_0035-3841_1966_num_64_83_1335

Fichier pdf généré le 25/04/2018 


\title{
Le fondamental est-il l'essentiel ? ${ }^{(\prime)}$
}

\author{
(Aristote, Métaphysique Z 3)
}

Les considérations que nous voudrions soumettre auront un caractère polémique. Cela vaut tout $d$ 'abord pour la première partie de notre exposé. Nous nous efforcerons de montrer que, selon Aris-

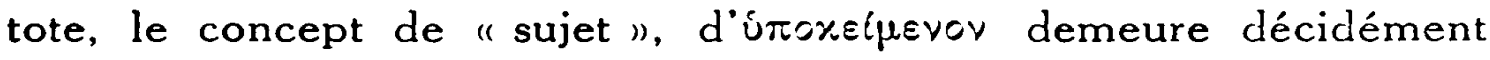
un concept insuffisant pour saisir l'essentiel de l'être des choses,

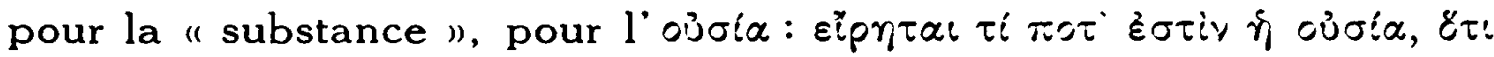

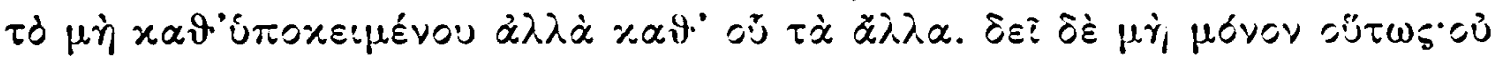

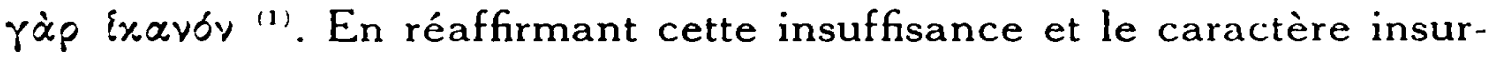
montable de celle-ci, nous nous opposerons de la façon la plus directe à une interprétation établie depuis longtemps, tout au moins depuis $S$. Thomas (encore que pour $S$. Thomas le problème se pose sous une forme originale). Mais ce n'est pas là la raison pour laquelle nous vous avertissons d'avance du caractère polémique de notre communication. Aussi n'y a-t-il personne dans ce cercle auquel nous avons l'honneur de nous adresser qui nous fera un reproche d'opposer une critique à des interprétations traditionnelles, fussent-elles défendues par les plus illustres auteurs et commentateurs de notre histoire. Si donc nous vous prévenons expressément du caractère polémique de nos considérations, c'est pour une autre raison. Nous parlons de polémique, et non seulement de critique. Et par polémique, on entend, sinon un excès de critique, du moins une critique poursuivie pour elle-même. Nous voudrions donc vous avouer que nous sommes tenté de croire effectivement que la vocation de la philosophie est, non pas tout à fait en ce sens peu précis, mais pourtant dans un sens qui pourrait sembler

(*) Texte d'un exposé présenté au Séminaire de philosophie ancienne et médiévale, organisé par le Centre De W'ulf-Mansion de l'Institut supérieur de Phi'o. sophie de Louvain (20 mars 1963).

(1) Métaphysique, Z 3, 1029 a 8-9. 
être celui-là, une vocation polémique. Nous n'entendons assurément pas par là que les philosophes auraient pour mission de chercher querelle à tout le monde, encore que ce n'est peut-être pas un hasard si tel paraît être son désir à beaucoup d'autres. Ce qui nous semble, c'est que la tâche de la philosophie ne peut s'accomplir sans quelque recours à une certaine violence. Assurément, à nouveau, il ne pourra s'agir ici que d'une violence verbale. Il s'agira de cet effort violent qu'il faut pour faire ressortir d'une position donnée ses ultimes conséquences, pour découvrir les axiomes sur lesquels s'appuie en dernière analyse telle orientation de l'esprit ou d'un travail de l'homme, pour explorer les possibilités extrêmes réservées au monde, à l'histoire et à l'humanité, pour poser des questions qui pourraient encore se poser " à la limite ". Et cette sorte d'extrémisme n'a-t-elle pas toujours été un caractère distinctif des grandes philosophies - à leur époque ? Nous nous bornerons à vous en donner un seul exemple : il semble aujourd'hui définitivement établi que les sciences de la nature, et en particulier la physique, se soient enfin engagées sur la bonne voie, et même sur la voie royale du vrai savoir, du moins en ce qui concerne, précisément, la connaissance de la nature. Que les triomphes des sciences modernes soient éventuellement acquis au prix d'un abandon et d'une perte de ce qui devrait faire le vrai objet d'une recherche de la nature, cette possibilité ne semble plus guère aujourd'hui être réelle, ne semble plus guère prêter matière qu'à des spéculations gratuites de philosophes. Précisément! Et nous croirions que c'est là en effet une tâche du philosophe, et qui ne saurait manifestement être celle d'aucun savant, que de revenir sans cesse par ses questions sur pareilles possibilités extrêmes, aussi minimes paraissent-elles, et aussi longtemps qu'elles ne s'avèrent pas définitivement exclues, et d'une certitude absolue (2). En effet, il y a des possibilités qui semblent et qui sont très peu probables, mais dont les conséquences seraient d'une portée extraordinaire si jamais elles s'avéraient, en dépit de tout, être des réalités. Or on ne peut insister sur la portée des possibilités de limite sans faire violence à tout ce qui les contredit, sans nécessairement exagérer, sans attaquer tout ce qui semble établi et qui l'est en effet, sans se lancer dans la polémique.

(2) Cf. notre article: Les sciences exactes et l'idéal husserlien d'un savoir rigoureux, dans Archives de Philosophie, 27 (1964), pp. 424-438. 
Voilà une bien étrange introduction pour un exposé relatif à une question d'interprétation d'Aristote. Mais le fait est, nous le disions déjà, que nous n'entendons consacrer qu'une première partie de cette communication à ce problème d'interprétation. Ce que nous voudrions y montrer, nous ne saurions de toute façon le prouver dans le cadre de cette conférence. Nous avons essayé d'en fournir une preuve détaillée dans une centaine de pages d'un travail plus étendu ${ }^{(3)}$. Ces choses-là ne sont pas à prouver par des conférences, mais doivent l'être par écrit, de telle sorte que le lecteur puisse examiner à l'aise et à loisir l'argument. Nous nous limiterons donc, en ce qui concerne cette question, à vous exposer notre thèse et à chercher à vous convaincre seulement que cette thèse $n^{\prime}$ 'est du moins pas absurde et que la question à laquelle elle répond vaut la peine d'être posée et méditée. Mais nous ferons cet exposé pour en venir à une autre question, et qui sera la question de la portée qu'il faudra reconnaître à la réponse qui sera finalement donnée à notre première question, cette question d'interprétation d'Aristote. Nous vous annonçons donc d'emblée cette autre question que nous poserons et qui sera celle-ci : quelle différence cela fera-t-il que de dire, soit que, selon Aristote, la notion d'Ujoxeífevov est décidé. ment insuffisante pour saisir l'essentiel de l'être d'une oủola, soit que, pour Aristote, cette notion d'útoxeífsvov demeure le concept adéquat de l'oủoía, à condition qu'on l'entende correctement ? Et nous chercherons à vous convaincre que seule la portée polémique de cette décision peut lui conférer un intérêt et un sens, qu'inversement, une nécessité de polémique philosophique exige que l'on accorde une importance capitale à cette décision, et enfin, que la réponse qu'on a donnée jusqu'ici à cette question d'interprétation est trop faite, elle, pour apaiser toutes les polémiques et pour éviter une alternative philosophique d'une brûlante actualité. Voilà donc la raison de cette introduction dont nous ne savons si elle a été trop longue ou trop brève. Venons-en à notre premier propos.

Dans la première partie de notre exposé, nous aurons donc à vous présenter, premièrement, une esquisse de l'interprétation tra-

19. Rudolf Вовнм, Das Grundlegende und das Wesentliche. Zu Aristoteles' Abhandlung a Ueber das Sein und das Seiende, (Metaphysik Z), La Haye, Nijhoff, 1965. 
ditionnelle du $3^{e}$ chapitre du VII ${ }^{e}$ livre de la Métaphysique d'Aristote, deuxièmement, une critique de cette interprétation usuelle, et troisièmement, les éléments d'une interprétation nouvelle de ce même texte.

Les moments de l'interprétation traditionnelle de $Z 3$ peuvent à peu près se déduire à partir du seul postulat qui exige qu Aristote ne peut vouloir contester sérieusement la détermination de la substance comme sujet, qui nous est familière, du $5^{\circ}$ chapitre des Catégories. Dans cette hypothèse, l'insuffisance de ce concept de sujet pour saisir ce qu'est une substance ne peut viser ce concept de sujet en tant que tel. Plus particulièrement, la conséquence fâcheuse qui découle d'après Aristote d'une telle conception de la substance comme sujet, et d'après laquelle seule la matière s'avérerait être substance, ne peut être considérée à strictement parler comme une conséquence qui découlerait nécessairement du concept de sujet pour la substance. Au contraire, il doit être évident d'avance, pour Aristote, que ce n'est point seulement la matière qui peut se présenter comme substance à titre de sujet. Pourtant cette conséquence doit être possible en quelque manière, bien qu'elle soit erronée. Elle doit donc reposer sur un malentendu relatif au concept de sujet. Ce malentendu, à son tour, doit être rendu possible par un manque de clarté immédiate dans ce concept de sujet. Et ce sera donc en ce manque de clarté immédiate que consistera l" insuffisance " apparente ou immédiate d'une conception de la substance comme sujet. Cette insuffisance ne signifiera qu'une nécessité de tirer suffisamment au clair la notion même de sujet si l'on veut obtenir de celle-ci un concept adéquat de ce qu'est la substance. Cette interprétation traditionnelle peut donc se caractériser par les cinq moments suivants qu'on peut en effet retrouver, implicitement ou explicitement, dans tous les commentaires qui la défendent :

$1^{\circ}$ Il est supposé qu'Aristote part, dans Z 3, d'une réaffirmation de la thèse des Catégories ou d'une thèse très proche de cette dernière, d'après laquelle la substance est essentiellement sujet.

$2^{\circ}$ Il est entendu que, lorsque Aristote, après avoir cité les

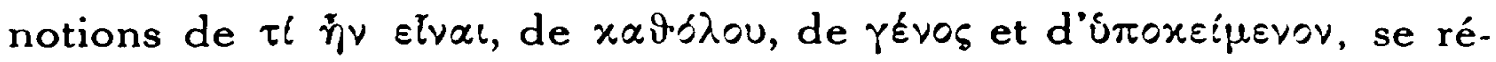
fère à la matière, à la forme et au composé des deux, il s'est déjà

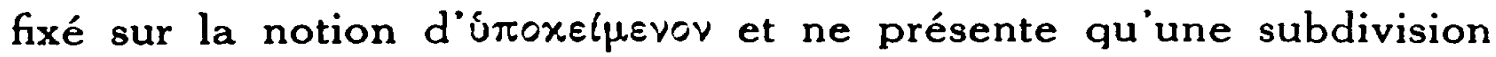
de cette dernière notion.

$3^{\circ}$ Il faut donc conclure que, lorsque Aristote développe ensuite, 
à partir de la conception de la substance comme sujet, la conséquence apparente d'après laquelle seule la matière serait substance à titre de sujet, il doit être entendu qu'il ne peut s'agir là que d'un malentendu relatif à la notion même de sujet.

$4^{\circ}$ On constate en effet qu'Aristote lui-même explique ce malentendu en soulignant le caractère "schématique " et le manque de " clarté " dont la notion de sujet reste entachée lors de sa première introduction. C'est sans doute en ces défauts précisément, et en eux seulement, que réside l'apparente insuffisance de la notion de sujet lorsqu'on veut saisir par elle la substance; ce sera donc chose aisée de réparer cette insuffisance immédiate.

$5^{\circ}$ On constate qu'en effet Aristote n'a point abandonné la notion de sujet pour caractériser la substance ; que la substance est et demeure pour lui un sujet, voilà qui est confirmé par de nombreux autres textes d'Aristote, en particulier par des textes dont il n'y a guère de doute qu'ils aient été écrits après celui du $3^{\circ}$ chapitre du livre $Z$ de la Métaphysique.

Nous passons tout de suite à un examen des arguments sur lesquels peut $s$ appuyer l'interprétation que nous venons d'esquisser. Nous nous référerons un à un aux moments énumérés.

$1^{\circ}$ Il est certainement contestable qu'Aristote part, dans Z 3 , d'une réaffirmation de la thèse des Catégories d'après laquelle la substance est essentiellement sujet. Aristote dit ici seulement :

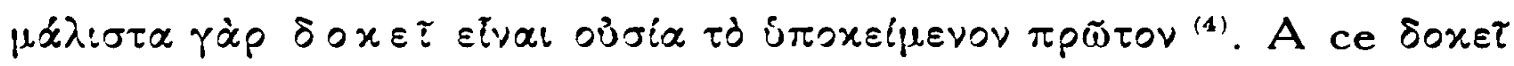

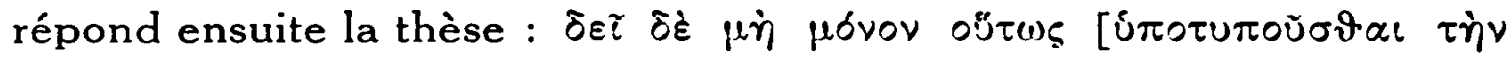

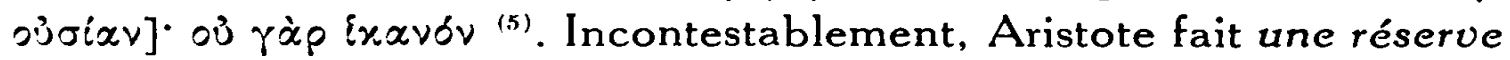
quant au caractère adéquat de cette conception de la substance. Toute la question est celle de savoir l'exacte portée de cette contestation. On ne peut limiter d'avance la portée de cette contestation en s'appuyant sur un simple renvoi aux Catégories : "it begs the question". Il faut du moins tenir compte de la possibilité qu'en fait Aristote entend précisément s'opposer à une conception de la substance telle qu'elle est exposée dans les Catégories. Et en effet, les commentateurs les plus circonspects parmi ceux qui soutiennent l'interprétation qui nous occupe ici, en particulier $M^{\text {110 }}$ Suzanne
(4) 1029 a $1-2$.
(3) 1029 a 9 (1028 b 31-32). 
Mansion 's), ont expressément renoncé à prendre appui d'avance sur un renvoi aux Catégories. Seulement, on peut se demander en outre si cette renonciation, à notre avis inévitable pour des raisons de rigueur méthodologique, ne prive pas d'emblée l'interprétation que nous discutons d'un appui indispensable. En effet, si l'on s'en tient au principe qui veut qu'un texte soit interprété de prime abord exclusivement à partir de lui-même, si on lit donc ce chapitre $3^{\text {n }}$ du livre $Z$ de la Métaphysique tel qu'il est écrit et sans recourir d'avance à des éléments extérieurs à lui, on ne peut y lire qu'une très explicite mise en question de la conception de la substance comme sujet, et on n'y trouve aucune conclusion qui ferait le poids de la démonstration de l'insuffisance de cette conception, démonstration qui est seule à répondre, dans le texte, à l'allégation initiale qui dit que "le sujet premier paraît être la substance, avec la plus grande apparence ". L“interprétation en cause semble donc nettement contrainte à faire recours à des éléments extérieurs au texte, tel le recours aux Catégories; mais elle devra justifier ce recours, et elle ne le pourra qu'en réussissant à établir une interprétation cohérente à partir de ce recours initial. Elle reposera donc entièrement sur les moments suivants que nous aurons à examiner. (Notons en passant ceci : en fait, comme cette interprétation ne peut se passer d'un recours à un élément étranger au texte lui-même, élément qui semble même, du moins à première vue, en contradiction expresse avec le contenu même du texte, cette interprétation devrait être précédée d'une démonstration de l'impossibilité de toute autre interprétation et qui s'appuierait exclusivement sur les données du texte même. En ce sens, on fait une importante concession à cette interprétation traditionnelle en lui accordant un examen préalable. Il suffrait, pour la critiquer, de montrer la possibilité d'une interprétation purement intrinsèque du texte, et ce serait l'affaire de ceux qui soutiennent l'interprétation traditionnelle de prouver l'incohérence de toute interprétation intrinsèque.)

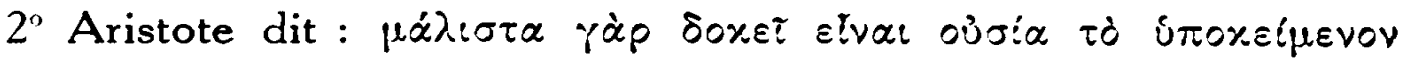

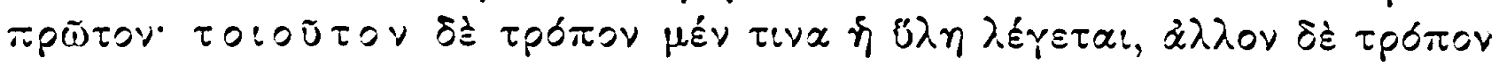

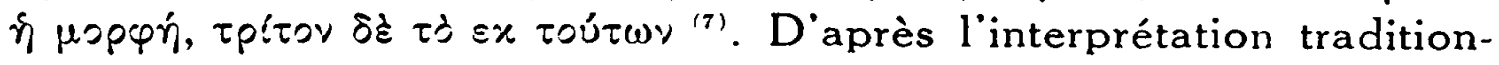

(6) Cf. Suzanne Mansion, La première doctrine de la substance: la substance sclon Aristote, dans la Revue philosophique de Louvain, 44 (1946), pp. 349-369.

(:) 1029 a $1-3$. 


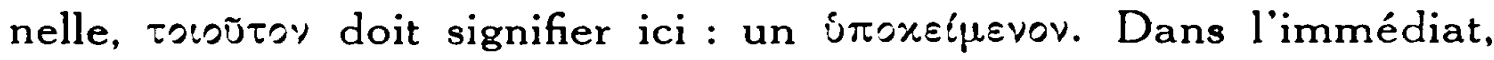
cette interprétation paraît certainement possible, et même naturelle. Les cornmentateurs se sont efforcés en particulier à démontrer qu'elle est aussi compatible avec la doctrine d'Aristote en général. Dans cet effort, ils ont rencontré certaines difficultés, en particulier à démontrer que la forme elle-même peut bien être considérée comme une sorte de sujet. Personnellement, nous ne voyons aucun texte qui le prouverait suffisamment. Mais la question n'est pas là. Il ne suffit pas d'établir que cette interprétation d'après laquelle Aristote décrit ici la matière, la forme et le composé comme trois sortes de sujets soit possible, il s'agit de savoir si cette interprétation est la bonne, si elle saisit bien ce qu'Aristote veut dire. Peut-on démontrer la nécessité de cette interprétation ? On ne le pourrait qu'en sappuyant sur l'affirmation qui constitue le premier moment de l'interprétation d'ensemble; mais nous avons vu que ce premier moment lui-même ne peut au contraire se confirmer que par la réussite de l'interprétation dans les moments suivants. Pour voir si cette interprétation du rscoũtov est la bonne, il est donc nécessaire de considérer la suite du texte. Or, dans la suite du texte, Aristote explique que la notion de sujet est ( $\mathrm{d}$ 'après nous) ou parait ( $\mathrm{d}$ 'après l'interprétation traditionnelle) - cela ne fait ici pas de différence - est ou tout au moins paraît donc être insuffisante, en faisant remarquer que cette conception conduit ou semble conduire à la conséquence inévitable que seule la matière est substance, et il dit : "Mais cela est impossible; car ... la forme et le composé semblent bien davantage être substance que la matière " ${ }^{(8)}$. L' $\{\pi 0$ -

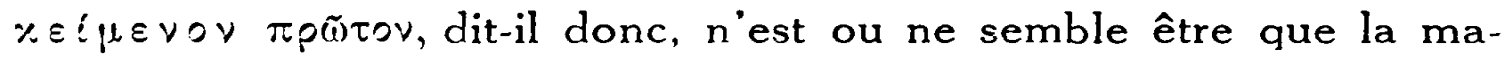
tière; mais la forme et le composé semblent sûrement être oủ $\sigma i \alpha \iota$ l'une et l'autre, et plus encore que la matière. Le critère auquel

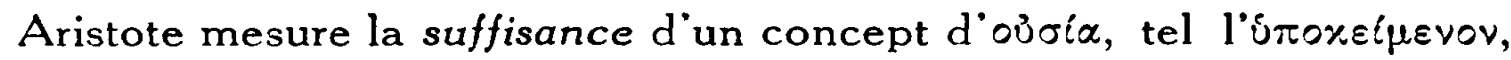
est donc constitué par l'exigence ou le présupposé qu'un concept adéquat de substance doit être capable de faire comprendre l'êtresubstance de la forme et du composé aussi bien que de la matière. Toute l'analyse d'Aristote repose donc sur ce présupposé : sont substance, la matière, la forme et le composé. Il serait donc étrange si Aristote n'énonçait pas expressément ce présupposé fondamental dès le début de son analyse, et si donc le tocoũ̃ov ne signifiait pas :

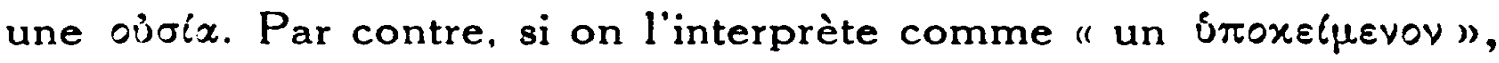


la confrontation de la notion de sujet elle-même avec le critère qui exige de toute notion de substance qu'elle soit capable de saisir l'être-substantiel de la forme, de la matière et du composé devient incompréhensible.

De plus, d'après l'interprétation traditionnelle, Aristote établirait donc d'abord nettement que le sujet, c'est la matière, la forme et le composé ; et ensuite, il affirmerait qu'un malentendu pourrait faire croire que seule la matière soit un sujet véritable; et enfin, il montrerait qu'il s'agit là d'un malentendu et d'une erreur en réaffirmant simplement que le sujet est bien les trois, et non seulement la matière (ce qu'il n'affirme du reste sûrement plus à la fin du texte d'une façon tant soit peu expresse). Il dirait donc que ne serait pas clair ce qu'il faut entendre par un sujet, après avoir clairement dit ce qu'il fallait comprendre par là. Nous voulons faire remarquer que l'interprétation traditionnelle semble ainsi en difficulté, pour suivre de près le Gedankengang effectif d'Aristote. Mais en fait, nous avons déjà abordé ici le troisième moment de cette interprétation. Il s'est manifesté, nous semble-t-il, que l'établissement du deuxième moment réclame un recours au troisième, comme déjà le premier moment nous renvoyait au deuxième et aux suivants. L“interprétation que nous discutons n’a pas encore réussi à prendre pied fermement dans le texte lui-même.

3" Aristote démontre de quelle façon on pourrait parvenir nous dirons : on ne peut ne pas parvenir -, à partir d'une conception de la substance comme sujet, à la conclusion qu'à ce titre de sujet seule la matière peut être retenue comme une véritable substance. D'après l'interprétation traditionnelle, ce raisonnement est erroné et repose sur un malentendu relatif à la notion de sujet. Pour réfuter cette interprétation, il suffit ici de citer Aristote. A aucun moment, en effet, il ne parle à ce propos de malentendu, d'erreur, de raisonnement erroné, d'illusion ou d'apparence. Il dit au contraire : le sujet premier paraît être la substance ${ }^{(8)}$. Mais ce concept de substance est insuffisant ${ }^{(10)}$. Car ainsi, la matière sera la substance ${ }^{(11)}$. Et encore une fois : lorsque l'on considère la substance comme le sujet, il est nécessaire que la matière se présente

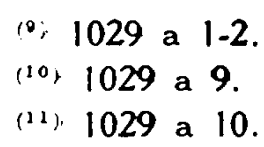


comme la seule substance ${ }^{(12)}$. Et encore une fois : dans une telle théorie, il arrive que la matière est la substance. Mais cela est impossible ${ }^{13)}$. Tout se passe doric comme si Aristote devinait déjà qu'on voudra croire qu'il entend simplement présenter un argument erroné dont il faudrait se garder, et comme s'il voulait faire comprendre par des affirmations fortement appuyées et plusieurs fois répétées qu'il s'agit bien d'une conséquence inévitable et absolument logique de la conception en cause, celle qui conçoit la substance comme sujet, qu'ainsi, seule la matière se présente finalement comme une substance véritablement substantielle.

Nous ne pouvons donc pas ne pas considérer que la théorie du "malentendu " qui constitue ce troisième moment de l'interprétation traditionnelle du texte, est une pure construction et une supposition qui ne peut $s^{\circ}$ appuyer sur aucun élément du texte luimême.

$4^{\circ}$ Mais s il n y a pas malentendu, s il n'est point question de raisonnement erroné, il est superflu et contradictoire de chercher dans le texte une explication de la possibilité du malentendu et de l'erreur. Le quatrième moment de l'interprétation traditionnelle consiste en ceci qu'elle interprète comme une telle explication du malentendu possible le fait qu'Aristote caractérise la notion d'úroxel-

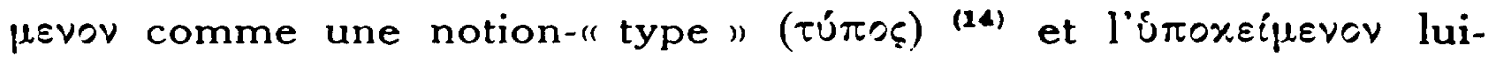

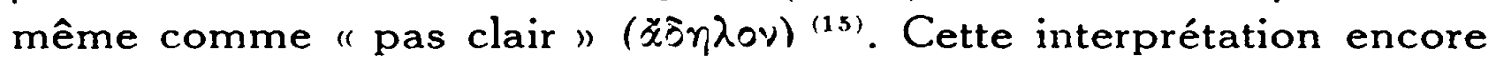
est arbitraire et $n^{\prime}$ 'a d'autre fondement que l'affirmation précédemment examinée par nous et d'après laquelle il faut penser à un malentendu possible qui aurait besoin d'explication. Que $\mathrm{l}^{\prime}$ ítoxєi-

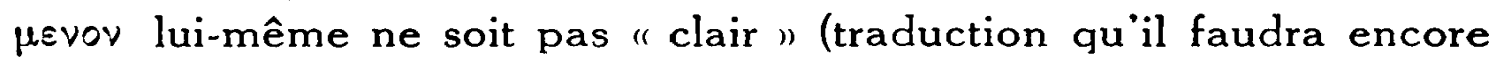
examiner de plus près). Aristote l'indique nettement comme une des raisons pour lesquelles il est insuffisant de concevoir la substance comme sujet; mais nullement comme une raison pour laquelle on puisse être induit dans l'" erreur " consistant à admettre, à partir de cette conception, que seule la matière se présente comme véritable substance. Or, comme c'est par référence à ce " pas clair"

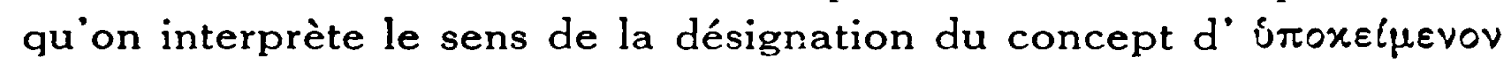

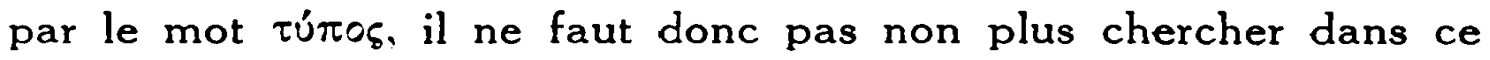

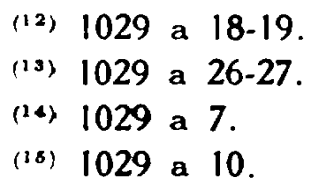




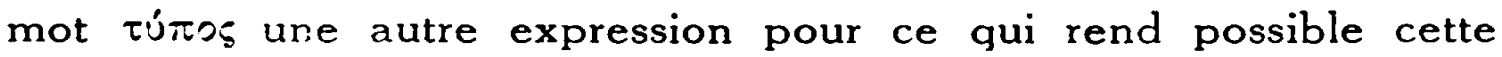
conséquence prétendument "erronée ". De toute façon, il est évident que l'ensemble de l'interprétation traditionnelle ne pourra être

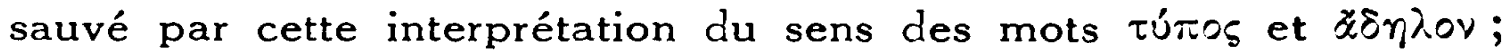
ici, la critique pourra vraiment se borner à montrer qu'une autre conception du sens de ces deux mots est certainement possible et plus directement suggérée par le texte. Nous ne nions cependant pas, tout au contraire, et nous voudrions même souligner qu'un

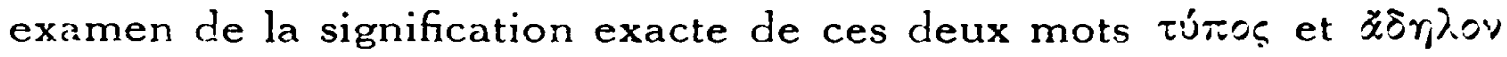
dans leur contexte conduira au centre même de toute la question du sens du chapitre $Z 3$ de la Métaphysique. Nous y reviendrons donc tout de suite lorsque nous exposerons les lignes générales d'une nouvelle interprétation de ce chapitre.

$5^{\circ}$ L interprétation traditionnelle s'appuie enfin sur un renvoi à d'autres textes d'Aristote où il continue certainement de considérer la substance comme un sujet. Il ne peut être question de nier ce fait. Seulement, si nos critiques précédentes paraissent tant soit peu justifiées, si donc l'interprétation nouvelle qui s'y est déjà annoncée semble probable, il s'agira de considérer s'il ne faut pas distinguer entre deux questions :

D'abord, toute substance est-elle un sujet, et seul un sujet peut-il être une substance ? A cette question, les autres textes $d$ 'Aristote auxquels on nous renvoie répondent nettement par l'affirmative, de sorte que le chapitre $Z 3$ ne peut pas y répondre négativement.

Mais il est une autre question : est-ce en tant que sujet que toute substance est - "essentiellement " - une substance ? Et nous affirmons que c'est à cette autre question, plus radicale, que répond le chapitre $Z 3$, et bien par la négative.

La récessité de distinguer entre ces deux questions simpose aisément. En effet, on peut admettre par exemple que tout homme est un animal, et que seul un animal peut être un homme. Mais il ne s'ensuit aucunement que c'est, "essentiellement" en tant qu'animal, que l'homme est homme. Il faudra cependant être plus précis encore. En effet, même si l'on a réussi à saisir un caractère absolument distinctif de l'être humain, à savoir qu'il est un animal raisonnable, on n'en est pas pour autant assuré d'avoir compris par là ce qui constitue l'essentiel de cet être. Cette question essentielle, le problème de l'essence, ne se réduit point à celle d'un signale- 
ment absolument univoque ou exhaustif. Par cette dernière remarque, nous passons à l'exposé de l'interprétation de $Z 3$ que nous voudrions opposer à l'interprétation traditionnelle.

Pour être bref, nous nous permettons d'exposer cette réinterprétation de manière dogmatique et sous la forme d'une paraphrase du texte d'Aristote.

Au chapitre $2^{n}$, qui précède. Aristote a énuméré toutes sortes de choses qui paraissent manifestement être des substances. Il a terminć cc chapitre en soulignant qu'on ne pourra toutefois décider en définitive lesquelles de ces choses sont effectivement des substances et lesquelles ne le sont pas, qu'après avoir d'abord répondu

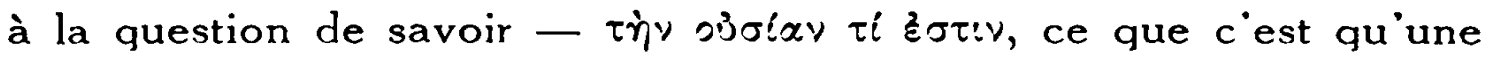
substance ${ }^{(16)}$. Puisque cette dernière question, qui sera celle des chapitres suivants, est aussi nettement opposée à celle de savoir

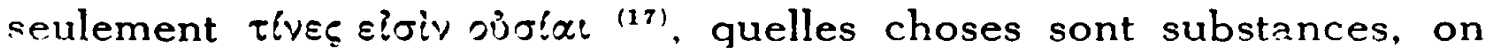
doit comprendre qu'il s'agira non seulement de fixer un signe distinctif valable pour toutes les substances (ce qui répondrait seulement à la question de savoir quelles choses sont substances), mais bien de chercher ce qui constitue l'essence même d'une substance en général. Déterminer ainsi l'essence même d'une substance en général, est ce qu'Aristote appelle dans les derniers mots du chanitre $2^{\circ}:$ : même nous indique en quel sens il parlera plus loin d'un concept

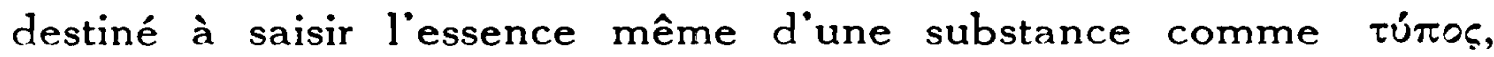

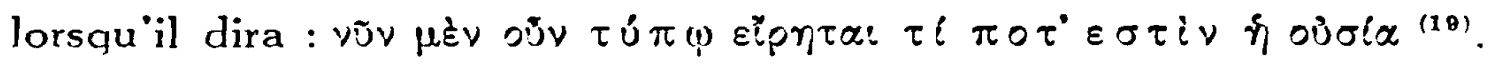

Aristote cite alors quatre notions qui pourraient répondre à la question touchant le "type " même d'une substance, c'est-à-dire ce qui caractérise "essentiellement" une substance en tant que

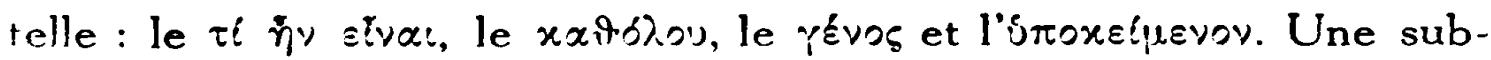

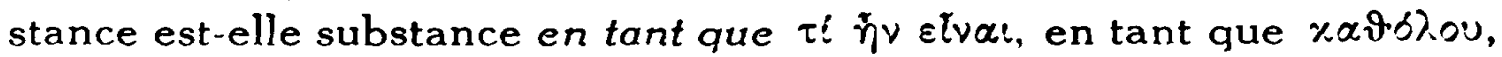

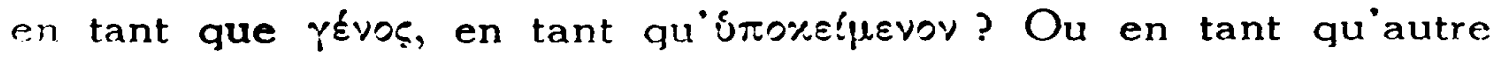

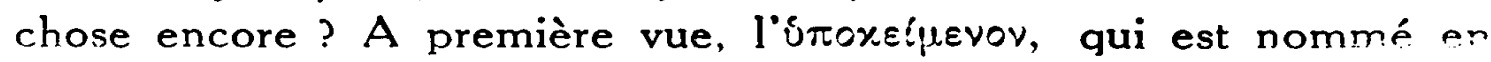
dernier lieu, semble le concept le plus adéquat; en effet, il vise directement ce à quoi tout concept de substance devra nécessairement être rapporté comme prédicat : faute de quoi, on substituerait

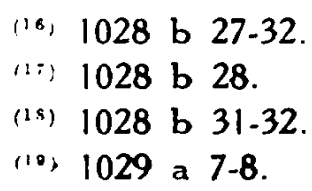


un tel concept à cela même qui est réellement une substance. Aristote décide donc d'examiner en premier lieu si ce concept d'ú $\pi \partial x \varepsilon \hat{l}$ levov est suffisant pour caractériser une substance en tant que telle. Avant de passer à cet examen, il énonce ce qui servira de critère pour un tel examen : les substances comprennent des matières, des formes, et des choses composées de forme et de matière. Cela est supposé manifeste. Un concept suffisant du "type " de la substance devra être capable de saisir l'être-substantiel des matières, des formes et des choses composées de forme et matière. Voilà donc ce qu'on venait d'envisager d'abord comme un tel

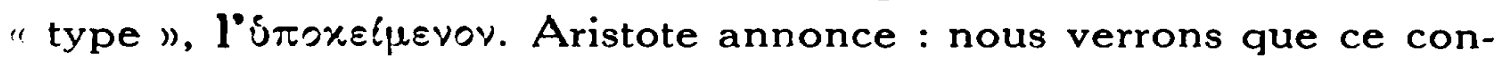
cept $n^{\prime}$ 'est pas suffisant, s'avérera insuffisant lorsqu'on le soumettra au critère indiqué. On constatera en effet, dit-il : premièrement,

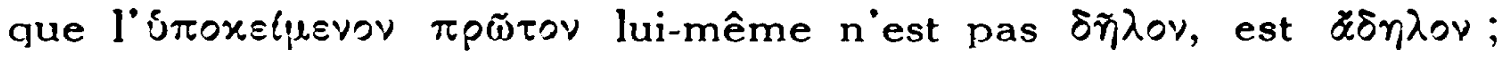
et deuxièmement, que l" identification" de substance et de sujet ne permettra que de comprendre l'être substantiel d'une matière.

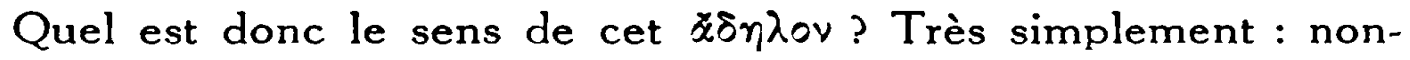
manifeste, pas manifeste. Abandonnons ici l'attitude dogmatique pour expliquer quelque peu les raisons qui nous suggèrent cette traduction. Aristote indique deux raisons pour lesquelles la conception de la substance comme sujet lui semble insuffisante, dont

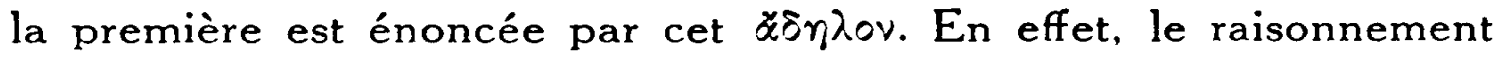
qui suit dans le texte aboutit à deux résultats, très nettement, bien que l'un et l'autre soient étroitement associés. Il montre d'abord

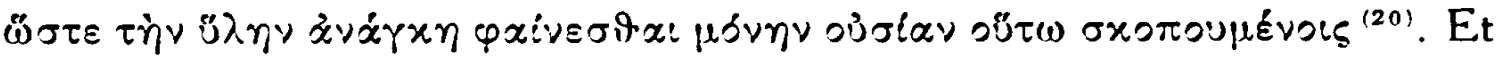
il ajoute qu'il s'agit d'une " matière qui en elle-même n'est à déterminer ni comme quelque chose ni comme une quantité ni en aucune autre des manières selon lesquelles un étant se définit... Ainsi donc, ce sujet dernier n'est en lui-même ni quelque chose ni une quantité ni rien d'autre " ${ }^{(21)}$. Il semble évident que cette précision touchant le point de savoir de "quelle" matière il s'agit ici, est à comprendre en opposition à cette autre description de la matière qu'Aristote donnait de celle-ci lorsqu'il énonçait auparavant quel sera le critère de l'examen : il parlait alors de la matière comme par exemple de l'airain dont est faite une statue ${ }^{(22)}$. Autrement dit, cette matière qui semble seule être une chose vraiment substantielle lorsqu' on considère que toute substance est substance en tant qu'elle
(20) 1029 a 18-19.
(21) 1029 a $20-25$.
(ग2) 1029 a 4. 
est sujet, n'est pas celle qui manifestement est quelque chose de substantiel. Cette " matière ", la prima materia, n'est même absolument pas quelque chose de manifeste, elle est essentiellement dépourvue de toute délimitation qui seule pourrait la rendre manifeste. La conception de la substance comme sujet conduit donc à poser comme la chose la plus substantielle ou même la seule chose véritablement substantielle, quelque chose qui, en soi, n'est point manifeste, et donc pas non plus manifestement une substance, mais qui n'est à poser comme substance qu'en vertu du raisonnement même qui découle du concept en question. Cette interprétation cadre parfaitement avec le rôle évident que joue dans tout ce texte, depuis le $2^{\mathrm{e}}$ chapitre déjà, une référence constante au $\delta \tilde{\eta} \lambda \circ \mathrm{v}$, au

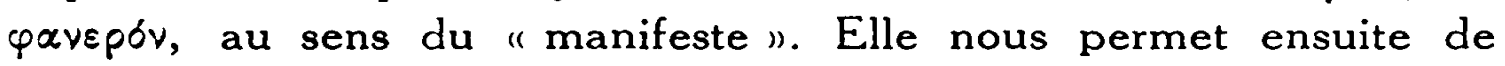
comprendre pourquoi Aristote reprend alors une deuxième fois la conclusion : " De tout cela, il résulte donc que c'est la matière qui est la substance " "2:3), pour continuer cette fois-ci : "Mais cela est impossible ; car ... la forme et le composé semblent bien davantage être substance que la matière "(24). C'est à présent seulement qu'il s'agit à proprement parler de la deuxième raison pour laquelle le concept de sujet est insuffisant pour caractériser l'essentiel d'une substance. Cette deuxième remarque est nécessaire, parce qu'on pourrait affirmer que si ce concept ne permet de reconnaître comme une substance au sens le plus radical de ce mot qu'un sujet dernier qui à proprement parler n'est "rien ", il permet toutefois de reconnaître un être substantiel à toute chose matérielle en tant qu'elle participe au caractère fondamental du "sujet dernier" ou de la " matière première ".

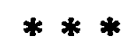

Posons ici une question. Quelle différence cela ferait-il finalement $s$ 'il fallait considérer que, pour Aristote, la notion de sujet est absolument insuffisante pour saisir l'essentiel d'une substance, ou que, selon Aristote, cette notion ne fournit un concept adéquat de la substance qu'à condition qu'on l'entende correctement ?

Dans ce dernier cas, si donc on soutient l'interprétation traditionnelle, on peut nous adresser, non sans quelque apparence 
d'avantage, le raisonnement suivant : vous estimez que la notion de sujet qui conduit à l'impossibilité de saisir par elle autre chose

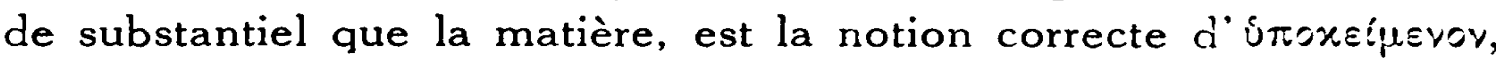
et vous croyez que telle est aussi l'opinion d'Aristote. Nous qui soutenons l'interprétation traditionnelle du texte, nous estimons au

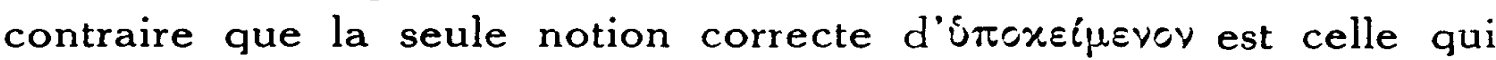
permet de saisir d'une façon adéquate ce qui est effectivement substance, et nous croyons que telle était aussi l'opinion du Stagirite. Mais nous serions même disposés à admettre éventuellement que la notion d'úroxsíf $\mu$ vov peut s'entendre correctement dans les deux sens : l'un, qui ne permet pas de désigner alors par ce concept la substance, et l'autre, qui est le seul sens correct lorsqu'on veut saisir adéquatement l'être d'une substance. Au fond, nous sommes donc d'accord ; finalement, seule une querelle de mots nous sépare, querelle qui porte, il est vrai, en premier lieu sur la question de savoir exactement ce qu'Aristote entendait ou voulait faire entendre

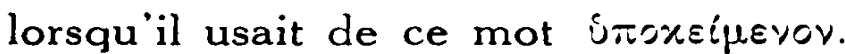

$\mathrm{Au}$ risque de paraître chercher à tout prix la querelle et la polémique, nous ne pouvons marquer notre accord. Nous ne le pouvons parce qu'il nous semble que trop souvent la réduction, presque toujours possible en matière de philosophie, d'une question litigieuse à une simple querelle de mots, menace de porter atteinte à la cause même de la philosophie. Avant toute autre chose, il faudrait reconnaître que notre problème touche surtout à la question suivante, posée par Aristote : qu'est-ce qu une chose substantielle ? c'est-à-dire, dans un sens radical : en quoi consiste l'essentiel dans tout ce qui est? C'est de cela qu'il s'agit de comprendre quelque chose et de pouvoir dire ce qu'on aura compris, c'està-dire d'en former un concept. Si l'on nous dit, par contre, qu'il ne s'agit que d'entendre correctement une notion telle que l' ínoxéffevov, on risque aussi bien de perdre de vue l'objet réel de la question que de renoncer à tout véritable concept : en effet, ne nous dit-on pas simplement que la notion d'íoxaífevov sera entendue correctement lorsqu'on entendra par elle, précisément, la substance ? Et cela ne revient-il pas à supposer qu'on aura déjà compris ce que c'est qu'une substance, sans concept, et donc à renoncer à ce concept, tout en se servant d'un terme qui doit suffire pour désigner ce qu'on entend désigner ? Sinon, il faudrait constater que W. D. Ross, par exemple, impute à Aristote un grossier sophisme lorsqu'il résume ainsi le raisonnement du cha- 
pitre Z $3:$ " ... the identification of substance with substratum tends to lead to the identification of it with matter... Thus the thought of substance as substratum leads to a wrong result. Instead of abandoning it, however. Aristotle ostensibly retains it, but infers that the substratum must be one of the other two things he had said it might be - form, or the unity of form and matter... But ... he here makes a fresh start; he leaves the notion of substratum and passes to another of the four original claimants of substantiality " (25).

Par contre, si l'on admet une interprétation telle que nous venons de l'esquisser, on est obligé de reconnaître que dans ce $3^{\circ}$ chapitre de son traité De l'être et de l'essence (26). Aristote ne se borne pas à rappeler une notion schématique et bien connue qui permet $d$ 'indiquer convenablement ce qu'est une substance et à prévenir un malentendu possible, mais il s'attaque de front à une question philosophique qui est aussi ancienne que la philosophie elle-même et qui aujourd'hui encore n'a rien perdu de son actualité, question formulée dans le titre de notre communication : le fondamental est-il l'essentiel?

Expliquons-nous. Dès l'abord de sa recherche thématique de ce qu'est cet étant essentiel qu'on appelle une substance, Aristote admet la priorité avec laquelle une conception a tendance à s'imposer à nous, d'après laquelle ce serait en tant qu' íroxeífrevov qu'une oujoi $\alpha$ est une ojo: $\alpha$. Si l'on croit avec nous que cette conception demeure néanmoins pour Aristote inadéquate, cette priorité devient quelque peu énigmatique. Cherchons donc à approfondir, avec l'aide d'Aristote lui-même, les raisons de cette priorité. Nous

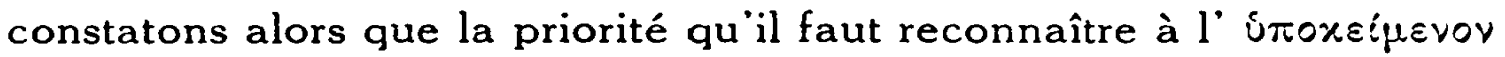
est très exactement celle qui se trouve définie ainsi par Aristote dans $\Delta \|$ : "On appelle antérieur ( $\pi \rho \delta \tau \varepsilon p o v)$ selon la nature et selon l'essence, ce qui peut être sans quelque chose d'autre, alors que cet autre ne peut être sans lui ; voilà un critère dont usait Platon " ${ }^{(27)}$. Il n'est en effet aucun besoin d'examiner si cette prio-

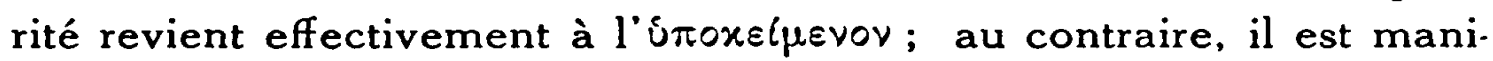

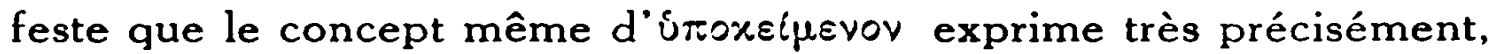
en dernière analyse, cette idée particulière de priorité. Dans les Analytiques postérieures (B 11), Aristote définit clairement I' i $\pi 0-$

(25) A ristotle's Metaphysics, Oxford, 3o éd., 1953, I, p. XcIv; les italiques sont de nous.

(28) Cf. 1053 b 17-18.

(27) 1019 a $2-4$. 
xéfuevov par la " nécessité qu'il soit, si quelque chose d'autre doit être " ${ }^{(28)}$. Il explique davantage cette idée dans des textes aussi importants que le chapitre final (9) de Physique B et le chapitre final (B 11) du De generatione et corruptione. Il l'explique par ailleurs de préférence par l'image d'une maison et de son fondement : sans ce fondement, la maison ne saurait être, alors que le fondement peut parfaitement être sans la maison, encore que par le fait même de l'existence d'un fondement, il n'y a pas encore

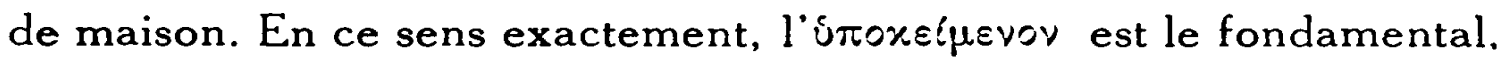

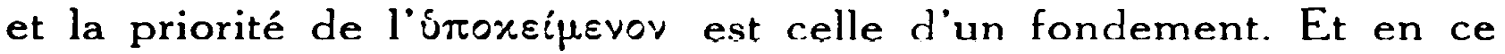
qui concerne le texte même de Métaphysique, $Z 3$, on constate sans difficulté - et on peut le confirmer par un renvoi aux considérations parallèles de Métaphysique, B 5-que le raisonnement qui y est exposé et qui doit montrer que l'identification de l'oủoía

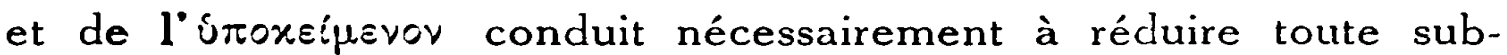
stance à la matière ${ }^{(29)}$, on constate que ce raisonnement est clairement basé sur l'idée de la priorité du fondamental au sens que nous venons de préciser.

C'est ainsi que dans ce chapitre Z 3, Aristote démontre : le fondamental est bien le fondamental, mais il n'est pas l'essentiel. L'essentiel, lui, est bien l'essentiel, mais il n'est pas le fondamental; il n'est fondamental que par rapport à autre chose, relativement, mais ce n'est pas en tant qu'il est fondamental qu'il est l'essentiel qu'il est.

On a toujours vu, certainement, que les considérations d'Aristote dans ce chapitre $Z 3$ contiennent une critique à l'égard de la pensée présocratique, et en particulier à l'égard du courant " matérialiste " de cette pensée. Il nous semble que la portée de cette critique est bien plus grande, tout comme la première conclusion du raisonnement présenté par Aristote, celle qui conduit à l'ăôn $\lambda \circ \%$, est plus radicale et plus importante encore que la deuxième, qui conduit à la matière comme seule substance. Nous nous demandons si Aristote ne vise pas par là, en effet, l'ensemble de la pensée grecque avant lui, Platon y compris. D'autre part, on sait que tout récemment Heidegger a formulé une nouvelle idée d'ontologie fondamentale qu'il a mise en rapport avec les conceptions originelles de la pensée présocratique. Or, il nous semble que les con-

(28) 94 a 21-23.

(20) 1029 a 11-19. 
sidérations critiques d'Aristote auxquelles nous nous référons atteignent les thèses heideggeriennes elles-mêmes. L'opposition heideggerienne à la pensée métaphysique qui, pour lui, va de Platon jusqu’à Nietzsche, peut en effet se résumer ainsi : l'essentiel de la métaphysique, ce que cette métaphysique tient pour l'essentiel, n'est pas le Fondamental - mais le fondement est un Etre qui a tous les traits de cet $\measuredangle \delta \eta \lambda$ ov dont nous parle Aristote. En ce sens, Aristote pourrait se déclarer d'accord avec Heidegger, sous la seule réserve qui toucherait l'emploi du mot Etre. Mais d'autre part, Heidegger voudrait manifestement maintenir en même temps que cet Etre fondamental dont il pose la différence par rapport à l'étant (la fameuse différence ontologique), est aussi l'Essentiel ; c'est pour cette raison précisément et en ce sens, sans doute, qu'il appelle ce fondement $\mathrm{l}^{\prime}$ "Etre ". C'est contre cette dernière prétention qu'Aristote nous fournit un argument de poids.

De plus, lorsque Heidegger s'efforce de montrer que cet is $\pi_{s-}$

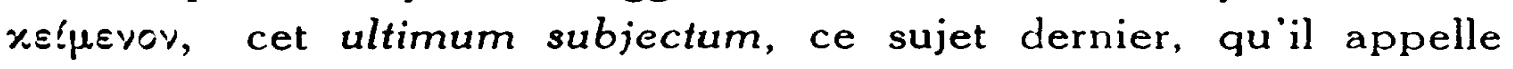
l'Etre "lui-même", est aussi l'essentiel de toute substance, il risque sans cesse de s'enliser dans une pensée dialectique qui repose. d'après les paroles de Hegel, sur la "conviction qu'il faut concevoir et exprimer le Vrai, non seulement comme substance, mais aussi bien comme sujet " ${ }^{(30)}$. Il vaudrait la peine de réexaminer à la lumière d'Aristote le sens ultime de cette notion hégélienne de " sujet", et du reste, plus généralement, celui de la subjectivité d'après laquelle on désigne la philosophie moderne comme "métaphysique de la subjectivité ".

Nous osons espérer que ces considérations auront contribué à mettre en lumière le rôle qui reste dévolu à la pensée et aux études aristotéliciennes dans le débat philosophique de nos jours.

Louvain.

Rudolf BOEHM.

(so) Phénoménologie de l'esprit, trad. par J. Hyppolite. Paris, s. d., 1, p. 17 (traduction modifiée par nous). 